\title{
Clinicopathological analysis of 114 cases of typical Kaposi's sarcoma in Xinjiang Uygur Autonomous Region, China
}

\author{
JUN-WEI FAN, XUE-FENG WAN, BIAN YI, JIN-CHENG DONG and PALIDA ABULIZE \\ Department of Dermatology, The First Affiliated Hospital of Xinjiang Medical \\ University, Urumqi, Xinjiang 830054, P.R. China
}

Received June 8, 2016; Accepted April 12, 2017

DOI: $10.3892 / \mathrm{mmr} .2017 .7283$

\begin{abstract}
The present study aimed to investigate the clinicopathological features of cases of classic Kaposi's sarcoma (CKS) in Xinjiang Uygur Autonomous Region, China, and analyze its etiology and treatment. A total of 114 patients, who were clinicopathologically diagnosed with CKS at the First Affiliated Hospital of Xinjiang Medical University (Urumqi, China) between 1980 and 2015 were retrospectively analyzed. The clinicopathological features of CKS were summarized, and its demographic distribution, pathogenesis, etiology and treatment were examined. The results revealed that, among the 114 patients with CKS, 100 patients were men and 14 patients were women, with a respective ratio of $7: 1$. The average age of these patients was 57.5 years old, and 97 of the patients were from the Uygur Autonomous Region (85.1\%). Among the 114 patients, 60 patients $(52.6 \%)$ were from Southern Xinjiang, 50 patients (43.9\%) were from Northern Xinjiang and four patients $(3.5 \%)$ were from Eastern Xinjiang. It was found that CKS in the Uygur ethnic group of Xinjiang Uygur Autonomous Region had unique clinicopathological features. The occurrence of CKS in Xinjiang may be associated with human herpes virus 8 infection, ethnicity-based susceptibility and lifestyle.
\end{abstract}

\section{Introduction}

Kaposi's sarcoma (KS), originally termed multiple idiopathic hemorrhagic sarcoma, is a malignant tumor, which was first described by Kaposi in 1782. Following the identification of acquired immune deficiency syndrome (AIDS) was identified by the United States Centers for Disease Control in 1980, KS was divided into four types in the majority of clinical practices: Classic, African, AIDS-related and iatrogenic (1).

Correspondence to: Dr Palida Abulize, Department of Dermatology, The First Affiliated Hospital of Xinjiang Medical University, 137 South Carp Hill Road, Xinshi, Urumqi, Xinjiang 830054, P.R. China

E-mail: abulizedoc@163.com

Key words: typical, Kaposi's sarcoma, clinical analysis, pathology
In China, the incidence of classic KS (CKS) in the Xinjiang region is high. In the present study, the clinicopathological data of 114 patients diagnosed with CKS at the First Affiliated Hospital of Xinjiang Medical University (Urumqi, China) were retrospectively analyzed. The details are reported as follows.

\section{Patients and methods}

The data of 114 patients who were clinicopathologically diagnosed with CKS at the First Affiliated Hospital of Xinjiang Medical University between 1980 and 2015 were collected and retrospectively analyzed. The inclusion criteria were as follows: i) patients of Chinese nationality and registered in the census as residents of the Xinjiang Uygur Autonomous Region; ii) diagnosed with CKS using clinicopathological methods; iii) only first visit data were analyzed from patients who visited the hospital a number of times. The exclusion criteria were as follows: i) foreign nationals or their registered permanent residence was not in Xinjiang; ii) patients with human immunodeficiency virus (HIV) infection, suspected HIV infection or positive results; iii) patients who had undergone renal transplantation and bone marrow transplantation. The data were described and processed using Microsoft Excel software version 2003 (Microsoft Corporation, Redmond, WA, USA).

\section{Results}

Evaluation of age of onset. Among all patients definitively diagnosed with CKS, disease onset in 13 patients $(11.3 \%)$ occurred during the period between 1980 and 1990; disease onset of nine patients $(7.8 \%)$ occurred during the period between 1991 and 2000; disease onset of 29 patients (25.2\%) occurred during the period between 2001 and 2010; and disease onset of 63 patients $(54.5 \%)$ occurred during the period between 2011 and 2015, as shown in Fig. 1.

General characteristics. Among the 114 patients with CKS, 100 patients were men and 14 patients were women, with a respective ratio of 7:1. The age of these patients ranged between 17 and 86 years old, with an average age of 57.5 years old. A total of 97 patients $(85.1 \%)$ were of the Uygur ethnic group, three patients $(2.7 \%)$ were of the Huis ethnic group, 13 patients (11.4\%) were Kazaks, and one patient (0.9\%) was Mongolian. 
On analysis of the geographical distribution of onset, 60 patients (52.6\%) were from Southern Xinjiang, 50 patients (43.9\%) were from Northern Xinjiang and four patients (3.5\%) were from Eastern Xinjiang. The distribution of patients from Southern Xinjiang were as follows: 31 patients from Kashi, 17 patients from Hotan, seven patients from Akesu, four patients from Kirghiz and one patient from Korla. The distribution of patients from Northern Xinjiang were: 18 patients from Urumqi, 16 patients from Yili, five patients from Changji, four patients from Aletai, three patients from Bozhou, two patients from Karamay and two patients from Tacheng. The four patients from Eastern Xinjiang were all from Turpan. The course of the disease ranged between 7 days and 22 years, and the average disease course lasted 2.7 years.

Clinical manifestations. In the 114 patients with CKS, disease in 112 patients (98.2\%) involved the extremities and 101 patients $(88.6 \%)$ exhibited a simple acromelic rash, in which two patients did not develop acromelic skin damage. In these two patients, disease was characterized by abdominal plaques in one patient, and by the development of neck and chest plaques in the other. A total of 70 patients (61.4\%) reported no notable symptoms, 36 patients $(31.6 \%)$ reported pain, and eight patients (7\%) developed itching.

All patients were clinically characterized by pink to purple erythema, plaques or nodules; and these erythema, plaques and nodules represent different stages of KS, namely the erythema stage, plaque stage and tumor stage. The rash during different these stages showed a spectrum of changes. In the erythema stage, it appeared as pink-purple patches of thumbnail size, and were either symptom-free or with marginal itching (Fig. 2A). As the course of the disease was prolonged, the color of the skin rash gradually deepened, and the patches transformed into plaques (Fig. 2B), which became purple nodules (Fig. 2C). For patients with severe conditions, the nodules fused into larger nodules or burst with accompanied pain.

Histopathological manifestations. The histopathological sections of the 114 patients were reviewed, which revealed that 14 patients $(12.3 \%)$ were in the erythema stage, 80 patients (70.2\%) were in the plaque stage and 20 patients $(17.5 \%)$ were in the tumor stage.

The patients in all three stages of disease revealed unique histopathological characteristics, which were consistent with clinical manifestations (Fig. 3). In the erythema stage, no notable anomalies were observed in the epidermis. Mild, strip-shaped hyperplasia of blood vessels was observed in the dermis. No deformed vascular endothelial cells were observed, and low levels of lymphocytes and plasma cells were shown to infiltrate the surroundings of blood vessels. The blood vessels were distributed in the epidermis in parallel (Fig. 3A and B).

In the plaque stage, strip-shaped hyperplasia was significantly increased. Various vascular cavity sizes were observed. The surrounding inflammation was more marked, with visible infiltration of eosinophilic spindle cells (Fig. 3C and D).

In the tumor stage, irregular fissure-like diffuse vascular hyperplasia was present in the dermis. A number of extravasated red blood cells were observed. Deformed vascular endothelial cells were significantly increased and a numerous eosinophilic spindle cell masses were observed (Fig. 3E and F).

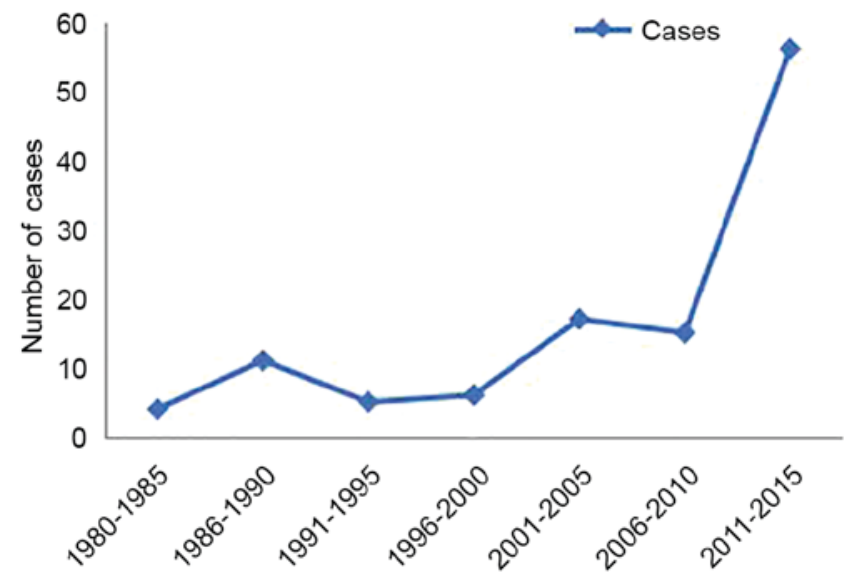

Figure 1. Distribution of age of onset of the 114 patients.

\section{Discussion}

CKS develops in immunocompetent and HIV-negative hosts of non-African origin, particularly among elderly men in the Mediterranean region. Human herpes virus 8 (HHV-8) or Kaposi's sarcoma-associated herpes virus (KSHV) has been demonstrated as causative agents for all types of $\operatorname{KS}(2,3)$. The seroprevalence of HHV-8 among the general population is estimated to be $>50 \%$ in sub-Saharan African countries, 20-30\% in the Mediterranean region, and $<10 \%$ in Northern Europe, Asia and the United States of America; and it has been closely correlated with the incidence of KS $(3,4)$. As HHV-8 is not detected in other vascular tumors, the detection of HHV-8 in spindle cell tumors is essential for diagnosing KS (3). HHV-8 is a major cause of the pathogenesis of $\mathrm{KS}$, which is present in 95\% of patients with KS, suggesting that HHV-8 has a leading role in the onset of KS (5). However, on a global scale, the infection rate of HHV-8 is significantly higher than the incidence of KS, suggesting that HHV-8 is necessary, but not sufficient, for the pathogenesis of KS. At present, the method of HHV-8 transmission remains to be elucidated. Previously, it was reported that sexual transmission, particularly between men, blood transfusion, and transmission by other body fluids, were likely to be the modes of infection of HHV-8 (6). However, in regions with a high incidence of CKS, no positive association between these methods of transmission and the incidence of SK has been found. Furthermore, studies investigating health workers in close contact with patients with KS did not demonstrate that the risk of HHV-8 infection was higher in this group, compared with the normal population (7). The specific immune mechanism underlying the induction of KS by HHV-8 infection also remains to be elucidated. KSHV induces the expression of angiogenic and inflammatory cytokines, which enables the virus to be transmitted to other adjacent cells, leading to vascular lesions and the formation of abnormal vascular spaces, which contain a mixture of inflammatory cells. KSHV can induce genomic instability, leading to mutations in cellular genes. Certain mutations, for example those in tumor protein 53 , can lead to further genomic instability and the accumulation of cellular gene mutations. When these provide a proliferative and/or survival advantage, cells containing these mutations may expand and lead to development of the oligoclonal or 
A

B

C

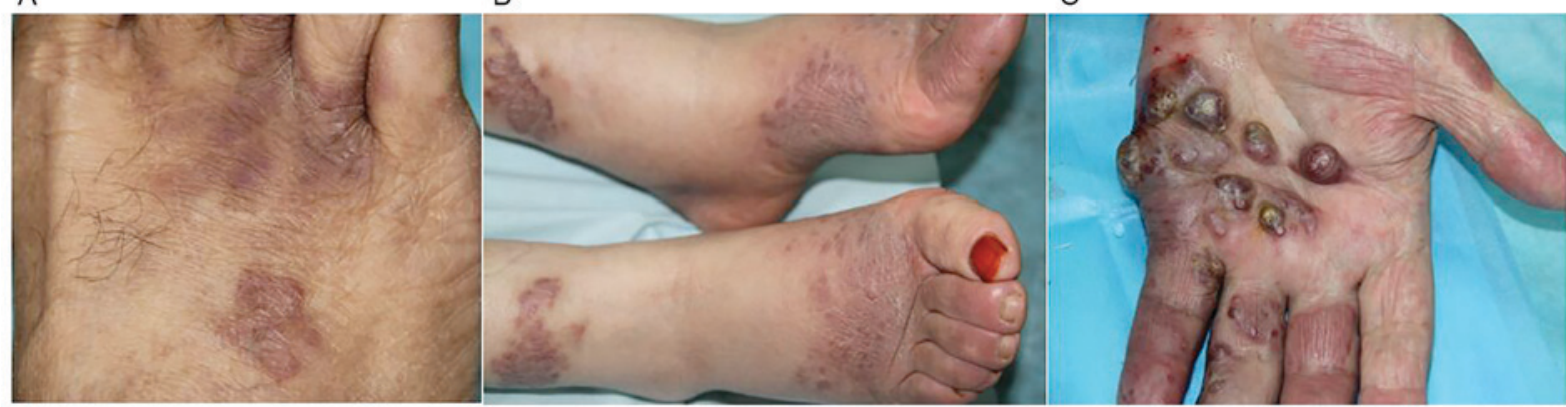

Figure 2. Typical clinical manifestations at different stages of disease. (A) KS at the erythema stage; (B) KS at the plaque stage; (C) KS at the tumor stage. KS, Kaposi's sarcoma.

A

B

C

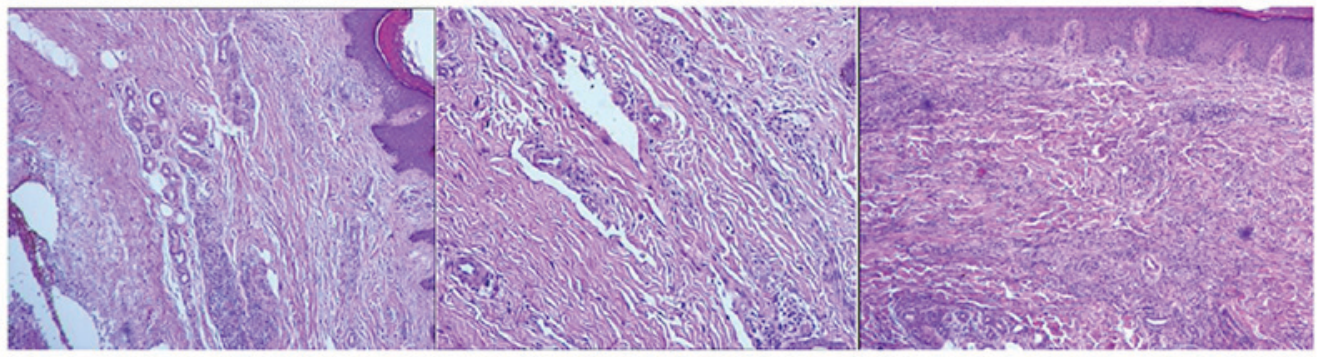

D

E

F

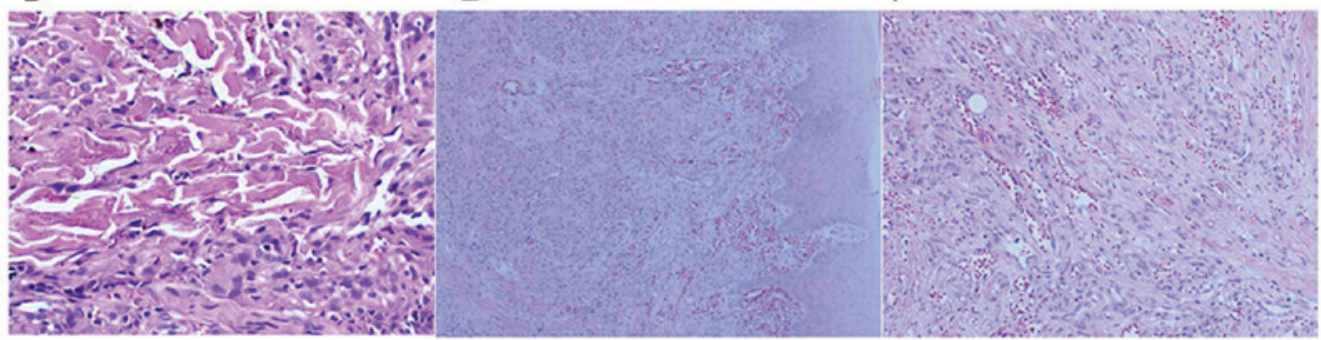

Figure 3. Typical pathological manifestations at different stages of disease. (A) KS at the erythema stage (HE; 10x4); (B) KS at the erythema stage (HE; 10x10); (C) KS at the plaque stage (HE; 10x4); (D) KS at the plaque stage (HE; 10x20); (E) KS at the tumor stage (HE; 10x4); (F) KS at the tumor stage (HE; 10x10). $\mathrm{KS}$, Kaposi's sarcoma; HE, hematoxylin and eosin..

(less commonly) the monoclonal expansion of neoplastic cells containing these driving mutations (8). HHV-8 infection and the involvement of various cytokines in the serum may constitute the common pathogenesis of CKS, with compelling data indicating the KSHV-encoded $\mathrm{G}$ protein-coupled receptor as a leading candidate viral gene for the initiation of KS (9). A study performed by Aka et al (10) revealed that increased serum levels of C-X-C motif chemokine 10, soluble interleukin (sIL)-1 receptor type II, sIL-2 receptor $\alpha$ and C-C motif chemokine ligand 3 may be important in the pathogenesis of $\mathrm{KS}$.

The data analyzed in the present study showed that the male-female ratio of $\mathrm{KS}$ incidence in Xinjiang was 7:1, which was lower than that in an Eastern European and Mediterranean origin report (15:1) (1). The fact that the incidence in men is higher compared with that in in women indicates that androgen levels are involved in the pathogenesis of CKS. However, further investigations are required to confirm this.

The data examined the present study revealed that the average onset age of CKS was 57.5 years old, and patients $>50$ years old were identified as the CKS-prone population.
The data did not reveal any pediatric cases. On a global scale, pediatric cases of CKS are rare. By 2011, only 30 cases of pediatric KS had been reported worldwide (11). The fact that the incidence of $\mathrm{KS}$ in children is low indicates the potential significance of hormone levels in the pathogenesis of KS.

According to statistical data from 2014, the number of Uygurs in Xinjiang was 10.5286 million. In this population, the incidence of CKS was $9.2 \%$. Despite the Han population of 8.4729 million in Xinjiang, there have been no cases of KS in the last 35 years. The Kazak population of 1.5575 million had an incidence of $8.3 \%$, the Hui population of 1.0231 million had an incidence of $2.9 \%$, and the Mongolian population of 0.1808 million had an incidence of $5.6 \%$. The difference in incidence was not statistically significant between the Uygur and Kazak ethnic groups; however, the differences in incidence were statistically significant among the Han, Uygur and Kazak ethnic groups. The Uygur and Kazak populations are the primary populations with KS in Xinjiang, whereas no CKS cases have been found in the Han population. This suggests the possibility of ethnicity-associated susceptibility to CKS. 
The incidence of KS also has endemic characteristics (12). In China, cases of CKS are concentrated in the Xinjiang region. In the Xinjiang region, there is no significant difference in the incidence distribution between Northern Xinjiang and Southern Xinjiang. The endemic characteristics were weak, which further confirms the ethnicity-associated susceptibility to CKS.

In 2008, Anderson et al (13) reported that smoking, diabetes and the use of glucocorticoids may be risk factors for the pathogenesis of CKS. The Uygur and Kazak populations in Xinjiang are Muslim, and Muslims tend not to smoke. In addition, the Uygurs and Kazaks in Xinjiang consume higher proportions of meat and starchy foods in their diet, associated with a high incidence of diabetes. These lifestyle factors may promote the occurrence and development of CKS in Xinjiang to a certain extent. Furthermore, naan bread is a common food eaten by the Uygur and Kazak populations in Xinjiang. This food is relatively dry, readily causing damage to the oral mucosa, and may be associated with HHV-8 infection (14). Therefore, the pathogenesis of CKS in Uygurs and Kazaks in Xinjiang may be caused by viral, tumor, immune and environmental factors.

CKS is likely to begin at the extremities. The data in the present study revealed that $98.2 \%$ of these patients had skin lesions involving the extremities. According to the course of the disease, CKS is characterized by reddish to purple erythema, and patches and nodules in the extremities; although the neck, chest and abdomen can be infected in a number of cases, for example, Errichetti et al (15) reported a case of CKS in the vulva in 2015. These data suggest that CKS primarily occurs in the extremities. However, it can affect other parts of the body in a limited number of patients.

The optimum treatment approach for $\mathrm{KS}$ depends on several factors, including the location of the lesions, disease progression, severity of symptoms and patient preference (16). The traditional treatment strategy comprises radiotherapy and chemotherapy, however, a single lesion or large nodules can be treated with surgical resection. Previous investigations have revealed that curettage followed by the application of $\mathrm{H}_{2} \mathrm{O}_{2}$ is a safe, effective and simple technique for the treatment of KS nodules in elderly patients (17). Vassallo et al (18) reported that the intralesional injection of vincristine can have sufficient curative effects against CKS in patients with diabetes. For patients showing poor therapeutic outcome from drugs, electrochemotherapy has been suggested as the 'new standard of care' as a first line treatment strategy for patients with stage I-II CKS (19). A previous study revealed that, as with the treatment of infant hemangioma, $\beta$-blockers can achieve a therapeutic effect in treating CKS (20). Another study reported that the external application of rapamycin, a mammalian target of rapamycin signaling pathway inhibitor, achieved a beneficial therapeutic effect (9). These techniques are likely to be the direction of further investigations in the treatment of CKS.

\section{References}

1. Antman K and Chang Y: Kaposi's sarcoma: N Engl J Med 342: 1027-1038, 2000.

2. Chang Y,Cesarman E, Pessin MS, Lee F, Culpepper J, Knowles DM and Moore PS: Identification of herpesvirus-like DNA sequences in AIDS-associated Kaposi's sarcoma. Science 266: 1865-1869, 1994.

3. Goldblum JR, Weiss SW and Folpe AL (eds): Kaposi sarcoma. Enzinger and Weiss's soft tissue tumors. 6th edition. Elsevier, Philadelphia, PA, pp723-730, 2014.

4. Nakajima T, Fujiwara M, Nishino Y, Kadowaki M, Shimamoto S, Fushimi H and Shimazu K: Case of classic Kaposi's sarcoma. J Dermatol 43: 834-835, 2016.

5. Greenblatt RM: Kaposi's sarcoma and human herpesvirus-8. Infect Dis Clin North Am 12: 63-82, 1998.

6. Pica F and Volpi A: Transmission of human herpesvirus 8: An update. Curr Opin Infect Dis 20: 152-156, 2007.

7. Mancuso R, Brambilla L, Boneschi V, Hernis A, Agostini S, Tourlaki A, Bellinvia $\mathrm{M}$ and Clerici M: Continuous exposure to Kaposi sarcoma-associated herpesvirus (KSHV) in healthcare workers does not result in KSHV infection. J Hosp Infect 85: 66-68, 2013.

8. Schulz TF and Cesarman E: Kaposi Sarcoma-associated Herpesvirus: Mechanisms of oncogenesis. Curr Opin Virol 14: 116-128, 2015.

9. Díaz-Ley B, Grillo E, Ríos-Buceta L, Paoli J, Moreno C, Vano-Galván S and Jaén-Olasolo P: Classic Kaposi's sarcoma treated with topical rapamycin. Dermatol Ther 28: 40-43, 2015.

10. Aka PV, Kemp TJ, Rabkin CS, Shiels MS, Polizzotto MN, Lauria C, Vitale F, Pinto LA and Goedert JJ: A multiplex panel of plasma markers of immunity and inflammation in classical kaposi sarcoma. J Infect Dis 211: 226-229, 2015.

11. Kalkan G, Akbay G, Gungor E, Eken A, Ozkaya O, Kutzner H and Eksioglu M: A case of classic Kaposi sarcoma in a 11-yearold male. Indian J Dermatol Venereol Leprol 77: 730, 2011.

12. Meireles P, Albuquerque G, Vieira M, Foia S, Ferro J, Carrilho C and Lunet N: Kaposi sarcoma incidence in Mozambique: National and regional estimates. Eur J Cancer Prev 24: 529-534, 2015.

13. Anderson LA, Lauria C, Romano N, Brown EE, Whitby D, Graubard BI, Li Y, Messina A, Gafà L, Vitale F and Goedert JJ: Risk factors for classical Kaposi sarcoma in a population-based case-control study in Sicily. Cancer Epidemiol Biomarkers Prev 17: 3435-3443, 2008.

14. Gong H, Pa L, Wang K, Mu H, Dong F, Ya S, Xu G, Tao N, Pan L, Wang B and Shan G: Prevalence of Diabetes and Associated Factors in the Uyghur and Han Population in Xinjiang, China. Int J Environ Res Public Health 12: 12792-12802, 2015.

15. Errichetti E, Stinco G, Pegolo E and Patrone P: Primary classic kaposi's sarcoma confined to the vulva in an HIV-negative patient. Ann Dermatol 27: 336-337, 2015.

16. Mirza YA, Altamura D, Hirbod T and Verdolini R: Long-term response of classic kaposi's sarcoma to intralesional doxorubicin: A case report. Case Rep Dermatol 7: 17-19, 2015.

17. Tourlaki A, Bellinvia M and Brambilla L: Recommended surgery of Kaposi's sarcoma nodules. J Dermatolog Treat 26: 354-356, 2015.

18. Vassallo C, Carugno A, Derlino F, Ciocca O, Brazzelli V and Borroni G: Intralesional vinblastine injections for treatment of classic Kaposi sarcoma in diabetic patients. Cutis 95: E28-E34, 2015.

19. Di Monta G, Caracò C, Benedetto L, La Padula S, Marone U, Tornesello ML, Buonaguro FM, Simeone E, Ascierto PA and Mozzillo N: Electrochemotherapy as 'new standard of care' treatment for cutaneous Kaposi's sarcoma. Eur J Surg Oncol 40: 61-66, 2014

20. Meseguer-Yebra C, Cardeñoso-Álvarez ME, Bordel-Gómez MT, Fraile-Alonso MC, Pérez-Losada ME and Sánchez-Estella J: Successful treatment of classic Kaposi sarcoma with topical timolol: Report of two cases. Br J Dermatol 173: 860-862, 2015. 